PROCEEDINGS OF THE

AMERICAN MATHEMATICAL SOCIETY

Volume 125, Number 1, January 1997, Pages 101-109

S 0002-9939(97)03645-9

\title{
ON INVERTIBILITY IN NON-SELFADJOINT OPERATOR ALGEBRAS
}

\author{
JUNXI ZHAO
}

(Communicated by Palle E. T. Jorgensen)

\begin{abstract}
Let $\mathcal{L}$ be a complete commutative subspace lattice on a Hilbert space. When $\mathcal{L}$ is purely atomic, we give a necessary and sufficient condition for $\sigma(T)=\sigma_{\mathcal{L}}(T)$ for every $T$ in alg $\mathcal{L}$, where $\sigma_{\mathcal{L}}(T)$ and $\sigma(T)$ denote the spectrum of $T$ in $\operatorname{alg} \mathcal{L}$ and $B(H)$ respectively. In addition, we discuss the properties of the spectra and the invertibility conditions for operators in $a \lg \mathcal{L}$.
\end{abstract}

\section{INTRODUCTION}

The invertibility of a nest algebra, the typical non-selfadjoint operator algebra, has been discussed by some authors (see $[2,4,8]$ ). Motivated by the invertibility in selfadjoint operator algebras and the problem of whether the invertible group of a nest algebra is path-connected, we are interested in the invertibility in a commutative subspace lattice (abbr. CSL) algebras.

For $C^{*}$ algebras $\mathcal{A}$ and $\mathcal{B}$ with $\mathcal{A} \subset \mathcal{B}$, it is well-known that $\sigma_{\mathcal{A}}(T)=\sigma_{\mathcal{B}}(T)$ for all $T$ in $\mathcal{A}$. But the analogue is not valid generally for non-selfadjoint operator algebras. We discuss the conditions on $\mathcal{L}$ for alg $\mathcal{L}$ to be inverse closed for a CSL $\mathcal{L}$, and give a necessary and sufficient condition on $\mathcal{L}$ under which alg $\mathcal{L}$ is inverse closed.

Since CSL algebras are not inverse closed generally, we discuss the properties of the spectra of operators in them and give an invertibility condition similar to that in [4] for CSL algebras.

In the following, let $B(H)$ be the set of all bounded linear operators on a complex separable Hilbert space $H$. If $\mathcal{L}$ is a commutative subspace(or projection) lattice in $B(H)$ which is complete and contains 0 and $I$, then we denote by $\operatorname{alg} \mathcal{L}$ the set $\{T \in B(H): P T P=T P$, for every $P \in \mathcal{L}\} ;$ alg $\mathcal{L}$ is called a CSL algebra corresponding to $\mathcal{L}$. When $\mathcal{L}^{\prime \prime}$, the von Neumann algebra generated by $\mathcal{L}$, is purely atomic, that is, $I$ equals the sum of minimal projections in $\mathcal{L}^{\prime \prime}$, we say $\mathcal{L}$ is a purely atomic CSL. For arbitrary $P, Q$ in $\mathcal{L}$ with $P>Q, E=P-Q$ is an interval of $\mathcal{L}$. Minimal interval projections are known as the atoms of $\mathcal{L}$. When $\mathcal{L}$ is purely atomic, every projection in $\mathcal{L}$ is the sum of all atoms dominated by it. The intervals of $\mathcal{L}$ are partially ordered by the relation $\prec$, where $E_{1} \prec E_{2}$ if and only if $E_{1} B(H) E_{2} \subseteq \operatorname{alg} \mathcal{L}$. The relation $\prec$ is related essentially to the structure of alg $\mathcal{L}$ (see [3]). It is easy to see that if $E, F$ are atoms of $\mathcal{L}$, then $E \prec F$ if and only if

Received by the editors May 17, 1995.

1991 Mathematics Subject Classification. Primary 47D25.

Key words and phrases. Commutative subspace lattice, spectrum.

(C)1997 American Mathematical Society 
for every $P$ in $\mathcal{L}, P \geq F$ implies $P \geq E$. We use this relation $\prec$ to determine the condition under which $\operatorname{alg} \mathcal{L}$ is inverse closed.

\section{INVERSE CLOSEDNESS}

For a CSL $\mathcal{L}$ and every $T$ in alg $\mathcal{L}$, if $T$ invertible in $B(H)$ implies that $T^{-1}$ belongs to $\operatorname{alg} \mathcal{L}$, we say that $\operatorname{alg} \mathcal{L}$ is inverse closed. In this section, we discuss the conditions under which $\operatorname{alg} \mathcal{L}$ is inverse closed. For convenience, we first give several lemmas.

Lemma 2.1. Suppose that $T$ is invertible in $B(H)$. Then $T$ is invertible in alg $\mathcal{L}$ if and only if $T P H=P H$ for every $P$ in $\mathcal{L}$.

Proof. Suppose that $T$ is invertible in alg $\mathcal{L}$. For every $P$ in $\mathcal{L}, P H=T\left(T^{-1} P H\right) \subseteq$ $T P H \subseteq P H$. So $T P H=P H$. The converse is clear.

Lemma 2.2 [4]. Suppose that $T$ is an operator on $H$ such that the restriction of $T$ to an invariant subspace of finite codimension is invertible. If $T$ has trivial kernel, then $T$ is invertible on $H$.

Lemma 2.3. Let $T$ be an invertible operator in $B(H), P$ and $Q$ be invariant projections of $T$ with $P<Q$.

(1) If $T Q H=Q H$ and $\left.(Q-P) T\right|_{(Q-P) H}$ is injective, then $T P H=P H$. In particular, if $Q-P$ is of finite dimension and $(Q-P) T(Q-P) H=(Q-P) H$, then $T P H=P H$.

(2) If $T P H=P H$ and $(Q-P) T(Q-P) H=(Q-P) H$, then $T Q H=Q H$.

Proof. (1) Let $\left(\begin{array}{cc}A & C \\ 0 & B\end{array}\right)$ be the matrix of $\left.T\right|_{Q H}$ with respect to $P H \oplus(Q-P) H$. For any vector $y$ in $P H$, there exist vectors $x_{1}$ and $x_{2}$ in $P H$ and $(Q-P) H$ respectively such that $\left(\begin{array}{cc}A & C \\ 0 & B\end{array}\right)\left(\begin{array}{l}x_{1} \\ x_{2}\end{array}\right)=\left(\begin{array}{l}y \\ 0\end{array}\right)$, since $T Q H=Q H$. So, $A x_{1}+C x_{2}=y$ and $B x_{2}=0$. Since $B=\left.(Q-P) T\right|_{(Q-P) H}$ is injective, $x_{2}=0$ and $A x_{1}=y$, that is, $\left.T\right|_{P H} x_{1}=y$. By the arbitrariness of $y$ we have $T P H=P H$.

If $(Q-P)$ is of finite dimension, then $\left.(Q-P) T\right|_{(Q-P) H}$ is injective, because $(Q-P) T(Q-P) H=(Q-P) H$. So the result follows. (2) can be proved similarly.

Lemma 2.4. Let $\mathcal{L}$ be a CSL, $T \in$ alg $\mathcal{L}$ an invertible operator and $\left\{P_{i}\right\}_{i=1}^{\alpha}$ a sequence of projections in $\mathcal{L}$ with $T P_{i} H=P_{i} H$ for all $i$. Then $T\left(\vee_{i} P_{i}\right) H=\left(\vee_{i} P_{i}\right) H$ and $T\left(\wedge_{i} P_{i}\right) H=\left(\wedge P_{i}\right) H$.

Proof. Let $P=\vee_{i} P_{i}$. Suppose that $T P H \subset P H$. Take a nonzero vector $f$ in $P H \ominus T P H$. Since $P \geq P_{i}$ for each $i, T P H \supset T P_{i} H=P_{i} H$. Thus we have $f \perp P_{i} H$ for each $i$, and hence $f \perp\left(\vee_{i} P_{i}\right) H$, that is, $f \perp P H$. Therefore $f$ must be zero, a contradiction. So it follows that $T\left(\vee_{i} P_{i}\right) H=\left(\vee_{i} P_{i}\right) H$.

Let $Q=\wedge P_{i}$. Then $Q H=\cap P_{i} H$. If $T Q H \subset Q H$, then there exists a nonzero vector $g \in Q H \ominus T Q H$. Since $Q H \subseteq P_{i} H$ and $T P_{i} H=P_{i} H$ for each $i$, there exists a vector $f_{i}$ in $P_{i} H$ for each $i$, such that $T f_{i}=g$. By the invertibility of $T, f_{1}=f_{i}$ for all $i$. So $f_{1} \in \cap P_{i} H$ and $(g, g)=\left(T f_{1}, g\right)=0$. This is impossible. Therefore $T\left(\wedge P_{i}\right) H=\left(\wedge P_{i}\right) H$.

The following theorem is our main result in this section.

Theorem 2.5. Let $\mathcal{L}$ be a purely atomic CSL on $H$. Then alg $\mathcal{L}$ is inverse closed if and only if the following conditions are satisfied: 
(1) there do not exist two infinitely dimensional atoms $E, F$ of $\mathcal{L}$ with $E \prec F$,

(2) there does not exist a sequence $\left\{E_{n}\right\}_{n=0}^{\infty}$ of atoms of $\mathcal{L}$ such that $E_{0} \prec E_{n} \prec$ $E_{n+1}$ or $E_{n+1} \prec E_{n} \prec E_{0}$ for every $n \geq 1, E_{n} \neq E_{m}$ for $n \neq m$ and every $E_{n}$, but $E_{0}$ is finite dimensional, and

(3) there does not exist a sequence $\left\{E_{n}\right\}_{n=-\infty}^{\infty}$ of finitely dimensional atoms of $\mathcal{L}$ such that $E_{n} \prec E_{n+1}$ for all $n$ and $E_{n} \neq E_{m}$ for $n \neq m$.

Proof. Suppose that alg $\mathcal{L}$ is not inverse closed. We prove that at least one of (1), (2) and (3) in the theorem does not hold.

To the contrary, we suppose that (1), (2) and (3) are all true. Take an operator $T$ in $\operatorname{alg} \mathcal{L}$ such that $T$ is invertible in $B(H)$ but $T^{-1}$ is not in $\operatorname{alg} \mathcal{L}$. Then there exists a projection $P_{0}$ in $\mathcal{L}$ such that $T P_{0} H \subset P_{0} H$. If there existed a sequence of atoms of $\mathcal{L},\left\{E_{n}\right\}_{n=-\infty}^{\infty}$, such that $E_{n} \prec E_{n+1}$ for all $n$ and $E_{n} \neq E_{m}$ for $n \neq m$, then it would be clear that one of (1), (2) and (3) in the theorem is not true. So, it follows that $\mathcal{L}$ has no such sequence of atoms. We show that there must exist at least one atom $E_{0}$ with $E_{0} T E_{0} H \subset E_{0} H$, that is, $E_{0} H \ominus E_{0} T E_{0} H \neq(0)$ if (2) in the theorem is true.

To do this, we suppose that, for any atom $E$ of $\mathcal{L}, E H=E T E H$. Then it is clear that $P_{0}$ is not an atom. For an atom $E$ of $\mathcal{L}$, if there does not exist a sequence $\left\{E_{n}\right\}_{n=1}^{\infty}$ of mutually different atoms such that $E \prec E_{n} \prec E_{n+1}\left(E \succ E_{n} \succ E_{n+1}\right)$ for $n \geq 1$, we say that $E$ is upper (lower) finite. Let $E_{0}<P^{\prime} \in \mathcal{L}$ be a lower finite atom. We show that $E_{0} H \subseteq T P^{\prime} H$ and $\left.E_{0} T\right|_{E_{0} H}$ is injective.

Let $P_{E_{0}}=\wedge\left\{P \in \mathcal{L}: P E_{0}=E_{0}\right\}$. It is clear that $P_{E_{0}} \leq P^{\prime}$. If $T P_{E_{0}} H \neq P_{E_{0}} H$, then it is easy to see that $P_{E_{0}}$ is not an atom of $\mathcal{L}$. Put $Q_{E_{0}}=\vee\left\{P_{E^{\prime}}: E^{\prime}\right.$ is an atom of $\mathcal{L}$ with $E^{\prime}<P_{E_{0}}$ and $\left.E^{\prime} \neq E_{0}\right\}$. It is obvious that $E_{0}=P_{E_{0}}-Q_{E_{0}}$. By Lemma 2.4 and Lemma 2.3, it follows that there exists at least one atom $E_{1}$ of $\mathcal{L}$ such that $E_{1} \neq E_{0}, E_{1}<P_{E_{0}}$ and $T P_{E_{1}} H \neq P_{E_{1}} H$. So $E_{0} \succ E_{1}$. Inductively, we can choose a sequence $\left\{E_{n}\right\}_{n=1}^{\infty}$ of mutually different atoms such that $E_{0} \succ E_{n} \succ E_{n+1}$ for each $n$. This contradicts the assumption on $E_{0}$. So $T P^{\prime} H \supseteq T P_{E_{0}} H=P_{E_{0}} H \supseteq E_{0} H$. Similarly, we can prove that $T Q_{E_{0}} H=Q_{E_{0}} H$. Then it follows that $\left.E_{0} T\right|_{E_{0} H}$ is injective for $T E_{0} H \cap Q_{E_{0}} H=\{0\}$.

Now let $K$ be the set of all the atoms $E$ such that $E$ is lower finite and $E \leq P_{0}$. Put $Q_{0}=\sum_{E \in K} E$. Then $P_{0} \geq Q_{0} \in \mathcal{L}$. Indeed, let $Q_{0}^{\prime}=\vee_{E \in K} P_{E}$. Then $P_{0} \geq Q_{0}^{\prime} \geq Q_{0}$ and $Q_{0}^{\prime} \in \mathcal{L}$. If $Q_{0}^{\prime}>Q_{0}$, then there exists some atom $E$ of $\mathcal{L}$ such that $E \leq Q_{0}^{\prime}-Q_{0}$. Hence there is at least one atom $E^{\prime}$ in $K$ such that $E \leq P_{E^{\prime}}$. So $E^{\prime} \succ E$ (since $E^{\prime} \succ P_{E^{\prime}}$ ). By the choice of $E^{\prime}$, it follows that $E \in K$. This is impossible. Hence $Q_{0}^{\prime}=Q_{0}$. By Lemmas 2.4 and 2.3, we have $T P_{0} \supseteq T Q_{0} H=Q_{0} H$ and $Q_{0}<P_{0}$. Indeed, if $T Q_{0} H \subset Q_{0} H$, then there is an atom $E_{1} \in K$ such that $P_{E_{1}}$ satisfies $T P_{E_{1}} H \subset P_{E_{1}} H$ by Lemma 2.4. Let $Q_{1} \in \mathcal{L}$ be such that $E_{1}=P_{E_{1}}-Q_{1}$. Since $E_{1} T E_{1} H=E_{1} H$, by Lemma 2.3, $T Q_{1} H \subset Q_{1} H$. From Lemma 2.4, there is some atom $E_{2} \leq Q_{1}$ such that $T P_{E_{2}} H \subset P_{E_{2}} H$. It is clear that $E_{1} \succ E_{2}$, because $E_{1} \succ P_{E_{1}}$. Inductively, we have a sequence $\left\{E_{n}\right\}_{1}^{\infty}$ of mutually different atoms of $\mathcal{L}$ such that $E_{1} \succ E_{2} \succ \cdots$. This contradicts the fact that $E_{1} \in K$.

Let $K^{\prime}$ be the set of all atoms $E$ of $\mathcal{L}$ with either $E P_{0}=0$ and there is some atom $E^{\prime}$ such that $E^{\prime} \leq P_{0}-Q_{0}$ and $E \succ E^{\prime}$, or $E$ is upper finite. Put $P_{1}=\wedge\{P \in \mathcal{L}$ : $P E=E$ and $P \geq P_{0}$ for any atom $\left.E \in K^{\prime}\right\}$. Then $T P_{1} H=P_{1} H$. Indeed, if not, let $f \in P_{1} H \ominus T P_{1} H \subset P_{1} H \ominus Q_{0} H$ and $g=T^{-1} f$. Then $g \notin P_{1} H$ and $Q_{0} f=0$. 
Let $P_{1}^{\prime}=\wedge\left\{P \in \mathcal{L}: P g=g\right.$ and $\left.P \geq P_{1}\right\}$. Since

$$
f=\left(P_{1}-Q_{0}\right) T\left(P_{1}^{\prime}-P_{1}\right) g+\left(P_{1}-Q_{0}\right) T P_{1} g
$$

we have

$$
\left(P_{1}-Q_{0}\right) T\left(P_{1}^{\prime}-P_{1}\right) g \neq 0 \text {. }
$$

Hence there must exist atoms $E_{1} \leq P_{1}-Q_{0}$ and $E_{1}^{\prime} \leq P_{1}^{\prime}-P_{1}$ such that $E_{1} T E_{1}^{\prime} g \neq$ 0 . So we can deduce that $E_{1}^{\prime} \succ E_{1}$. By the assumption and the choice of $K^{\prime}$, it is not hard to show that $E_{1}^{\prime} \in K^{\prime}$ and $E_{1}^{\prime}<P_{1}$, a contradiction.

Let $G$ be the set of all atoms $E$ with $E \leq P_{1}-P_{0}$. For an atom $F \in G$, if there exists an atom $E^{\prime} \leq P_{0}-Q_{0}$ such that $F \succ E^{\prime}$, then $F$ must be of finite dimension since, for each atom $E \leq P_{0}-Q_{0}$, there is a sequence $\left\{E_{n}\right\}_{n=1}^{\infty}$ of atoms such that $E \succ E_{n} \succ E_{n+1}$ for all $n$ and (2) in the theorem is true, and consequently $\left.F T\right|_{F H}$ is injective for $F T F H=F H$; if $F$ is lower finite then $\left.F T\right|_{F H}$ is injective. Thus, $\left.E T\right|_{E H}$ is injective for any $E \in G$. By the assumption, $G$ has some atom $E$ such that there does not exist another atom $E^{\prime}$ of $\mathcal{L}$ with $E^{\prime} \succ E$ and $E \succ E_{1}$ for some atom $E_{1} \leq P_{0}-Q_{0}$. Let $U_{1}=\left\{E_{11}, E_{12}, \cdots\right.$, $\left.E_{1 \alpha_{1}}\right\}$ ( $\alpha_{1}$ is an ordinal) be the set of all such atoms. Put $Q_{11}=P_{1}-E_{11}$; it is not hard to prove that $Q_{11} \in \mathcal{L}$. Since $E_{11} T E_{11} H=E_{11} H$ and $\left.E_{11} T\right|_{E_{11} H}$ is injective, we have $T Q_{11} H=Q_{11} H$ and $Q_{11} \geq P_{0}$ by Lemma 2.3. Again, let $Q_{12}=\left(P_{1}-E_{11}\right)-E_{12}=Q_{11}-E_{12}$. Similarly, it follows that $T Q_{12} H=Q_{12} H$ and $Q_{12} \geq P_{0}$. Continuing this process, we obtain $Q_{11}, Q_{12}, \cdots, Q_{1 \alpha_{1}} \in \mathcal{L}$ such that $T Q_{1 i} H=Q_{1 i} H$ and $Q_{1 i}>Q_{1 i+1} \geq P_{0}$. By Lemma 2.4, $P_{2}=\wedge_{i} Q_{1 i}$ satisfies the conditions: $T P_{2} H=P_{2} H$ and $P_{2} \geq P_{0}$. If $G-U_{1}=\emptyset$, we obtain that $P_{0}=P_{2}$ and the desired contradiction: $T P_{0} H=P_{0} H$. If $G-U_{1} \neq \emptyset$, as above we know that there is some atom $E \in G-U_{1}$ such that there exists some atom $E^{\prime}$ only in $U_{1}$ with $E^{\prime} \succ E$. Let $U_{2}=\left\{E_{21}, E_{22}, \cdots, E_{2 \alpha_{2}}\right\}$ be the set of all such atoms in $G$. As above, let $Q_{21}=P_{2}-E_{21}$. Then one can deduce similarly that $Q_{21} \in \mathcal{L}, T Q_{21} H=Q_{21} H$, $Q_{2 i+1}=P_{2}-\left(E_{21}+E_{22}+\cdots+E_{2 i}\right) \in \mathcal{L}$ and $T Q_{2 i+1} H=Q_{2 i+1} H$ for each $i$. Put $P_{3}=\wedge_{i} Q_{2 i}$. It follows that $T P_{3} H=P_{3} H$ and $P_{3} \geq P_{0}$ by Lemma 2.4. If $G-\left(U_{1} \cup U_{2}\right)=\emptyset$, then we have $P_{3}=P_{0}$ and $T P_{0} H=P_{0} H$, the desired contradiction. If $G-\left(U_{1} \cup U_{2}\right) \neq \emptyset$, we can continue this process as above and obtain a sequence $\left\{U_{i}\right\}$. If $G-\left(\cup_{i} U_{i}\right)=\emptyset$, then $\wedge P_{i}=P_{0}$ and we can obtain $T P_{0} H=P_{0} H$, the desired contradiction. If $G-\left(\cup_{i} U_{i}\right) \neq \emptyset$, then there must exist some atom $E^{\prime} \in G$ such that that there is some atom $E$ only in $\cup U_{i}$ with $E \succ E^{\prime}$ by the assumption. In the same way, we can continue the above process to obtain a countable set $\left\{P_{i}\right\} \subseteq \mathcal{L}$ such that $T P_{i} H=P_{i} H$ and $P_{i} \geq P_{0}$ for every $i$. Let $\left\{P_{i}\right\}_{i \in \Lambda}$ be the maximal set of such projections in $\mathcal{L}$. So we have $P_{0}=\wedge P_{i}$ and $T P_{0} H=P_{0} H$, the desired contradiction. Thus it follows that (2) in the theorem is not true or there is some atom $E_{0}$ such that $E_{0} T E_{0} H \neq E_{0} H$.

Suppose that (2) is true, and choose an atom $E_{0}$ with $E_{0} T E_{0} H \neq E_{0} H$. Let $P_{0}^{\prime}$ be the smallest element in $\mathcal{L}$ which dominates $E_{0}$. It follows that $T P_{0}^{\prime} H \subset P_{0}^{\prime} H$. Indeed, suppose that $T P_{0}^{\prime} H=P_{0}^{\prime} H$. Let $Q$ be in $\mathcal{L}$ with $E_{0}=P_{0}-Q$. For a nonzero vector $f \in E_{0} H \ominus E_{0} T E_{0} H$, we can choose a vector $g \in P_{0}^{\prime} H$ so that $T g=f$. Thus, $\|f\|^{2}=(f, f)=(T g, f)=\left(T E_{0} g+T Q g, f\right)=\left(T E_{0} g, f\right)+(Q T Q g, f)=0$, a contradiction. So $T P_{0}^{\prime} H \subset P_{0}^{\prime} H$.

Now choose a nonzero vector $f_{0}$ in $E_{0} H \ominus E_{0} T E_{0} H$. Then there exists some vector $f$ such that $T f=f_{0}$. It is easy to know that $f \notin P_{0}^{\prime} H$. Since $\mathcal{L}$ is purely atomic, there is a subset $\left\{E_{k}\right\}$ of atoms of $\mathcal{L}$ such that $f=\sum_{k} E_{k} f, E_{k} f \neq 0$ for all 
$k$. Thus $f_{0}=T f=\sum_{k} T E_{k} f$. So there is at least one $E_{k_{1}}$ such that $E_{0} T E_{k_{1}} f \neq 0$ and $E_{k_{1}} P_{0}^{\prime}=0$ for $f \notin P_{0}^{\prime} H$. Thus $E_{0} \prec E_{k_{1}}$. Further assume that every atom $E$ of $\mathcal{L}$ with $E_{0} \prec E$ is finite dimensional. If $E_{k_{1}} H \ominus E_{k_{1}} T E_{k_{1}} H=(0)$, then $E_{k_{1}} T E_{k_{1}} f \neq$ 0 since $E_{k_{1}}$ is of finite dimension, and thus $\left.E_{k_{1}} T\right|_{E_{k}} H$ is invertible. Since $E_{k_{1}} f_{0}=$ $\sum_{k} E_{k_{1}} T E_{k} f=0$, we have $E_{k_{1}} T E_{k_{1}} f=-\sum_{k \neq k_{1}} E_{k_{1}} T E_{k} f$. Therefore there is some $k_{2}\left(\neq k_{1}\right)$ such that $E_{k_{1}} T E_{k_{2}} f \neq 0$, and then $E_{k_{1}} \prec E_{k_{2}}$. If $E_{k_{1}} H \ominus$ $E_{k_{1}} T E_{k_{1}} H \neq(0)$, then we can find another $E_{k_{2}}\left(k_{1} \neq k_{2}\right)$ such that $E_{k_{1}} \prec E_{k_{2}}$ as above. So, by induction, we can find some infinite-dimensional atom $E_{1}$ of $\mathcal{L}$ such that $E_{0} \prec E_{1}$ or a sequence $\left\{E_{k}\right\}_{k=1}^{\infty}$ of finite dimensional atoms of $\mathcal{L}$ such that $E_{k} \prec E_{k+1}$ for $k \geq 0$ and $E_{n} \neq E_{m}$ for $m \neq n$.

If $E_{0}$ is infinite dimensional, then since (2) is true, (1) is not valid by above arguments. So, without loss of generality, assume that $E_{0}$ is finite dimensional. Let $P_{E}=\wedge\{P \in \mathcal{L}: P E=E\}$ for every atom $E$ of $\mathcal{L}$, and $E_{0}=P_{E_{0}}-Q$ for some $Q \in \mathcal{L}$. By the choice of $P_{E_{0}}$, we have $Q \prec E_{0}$. If $T Q H=Q H$, then we have $\left.T\right|_{P_{E_{0}} H}$ is invertible by Lemma 2.2. This is impossible for $T P_{E_{0}} H \subset P_{E_{0}} H$. So $T Q H \subset Q H$. If $Q$ is an atom of $\mathcal{L}$, then $Q$ is of infinite dimension and (1) or (2) in the theorem cannot hold. Hence $Q$ is not an atom. If $T P_{E^{\prime}} H=P_{E^{\prime}} H$ for any atom $E^{\prime}<Q$, then $T Q H=Q H$ by Lemma 2.4. This is impossible. Hence there must exist an atom $E_{1}^{\prime} \leq Q$ such that $T P_{E_{1}^{\prime}} H \subset P_{E_{1}^{\prime}} H$ and $E_{0} \succ E_{1}^{\prime}$. Inductively, we can find an infinite dimensional atom $E_{1}^{\prime}$ with $E_{1}^{\prime} \prec E_{0}$ or a sequence $\left\{E_{k}^{\prime}\right\}_{k=1}^{\infty}$ of finite dimensional atoms such that $E_{k+1}^{\prime} \prec E_{k}^{\prime} \prec E_{0}$ for all $k \geq 1$ and $E_{n}^{\prime} \neq E_{m}^{\prime}$ for $n \neq m$. Therefore combining the above arguments, it follows that one of (1) or $(3)$ is not valid. Thus we have proved that if $\operatorname{alg} \mathcal{L}$ is not inverse closed, then one of (1), (2) and (3) of the theorem does not hold.

For the necessity, suppose that one of (1), (2) and (3) is not satisfied. We show that there is an invertible operator $T \in \operatorname{alg} \mathcal{L}$ such that $T^{-1}$ is not in $\operatorname{alg} \mathcal{L}$. We only prove this for (3), since the others can be proved similarly and are omitted.

Let $\left\{E_{n}\right\}_{n=-\infty}^{\infty}$ be a sequence of atoms of $\mathcal{L}$ such that $E_{n} \prec E_{n+1}$ for each $n$ and $E_{n} \neq E_{m}$ for $n \neq m$. Choose a unit vector $e_{n}$ in $E_{n} H$ for every $n$. Define an operator $T$ :

$$
\begin{aligned}
& T e_{n+1}=e_{n}, \quad \text { for each } n, \\
& T x=x, \quad \text { for every } \quad x \in\left[\vee\left\{e_{n}: n \in \mathbb{Z}\right\}\right]^{\perp} .
\end{aligned}
$$

It is clear that $T$ is invertible in $B(H)$. Let $P$ be an arbitrary element in $\mathcal{L}$; if $P E_{n}=0$ or $P E_{n}=E_{n}$ for all $n$, then it is easy to see that $T P H=P H$. So suppose that there exists some $n$ such that $E_{n} P=E_{n}$ and $E_{n+k} P=0, k \geq 1$. Hence $P E_{k}=E_{k}$ for $k \leq n$. For every $x$ in $P H$, we write $x=x_{0}+\sum_{n}\left(x, e_{n}\right) e_{n}$, $x_{0} \in P H \ominus \sum_{k \leq n} E_{k} H$. Then

$$
T x=T x_{0}+\sum_{k \geq n+1}\left(x, e_{k}\right) T e_{k}=x_{0}+\sum_{k \geq n+1}\left(x, e_{k}\right) e_{k-1} \in P H .
$$

Hence $T \in \operatorname{alg} \mathcal{L}$. However, it is obvious that $T^{-1} \notin \operatorname{alg} \mathcal{L}$. The proof is complete.

Remark. Although Theorem 2.5 requires a CSL to be purely atomic, it is easy to see from the above proof that if there is some purely atomic sublattice $\mathcal{L}_{1}$ of $\mathcal{L}$ such that $\mathcal{L}_{1}$ does not satisfy the conditions of Theorem 2.5 with respect to the relation $\prec$ determined by $\mathcal{L}$, then alg $\mathcal{L}$ is not inverse closed. Also by Theorem 2.5 , it is clear that a CSL algebra on a finite dimensional space is always inverse closed. 
Corollary 2.6. If alg $\mathcal{L} E$ is not inverse closed for some interval $E$ of $\mathcal{L}$, neither is alg $\mathcal{L}$. If there exists a sublattice $\mathcal{L}_{1}$ of $\mathcal{L}$ such that $\mathcal{L}_{1}$ does not satisfy the conditions of Theorem 2.3 with respect to the partial order $\prec$ determined by $\mathcal{L}$, then alg $\mathcal{L}$ is not inverse closed.

Corollary 2.7. Let $\mathcal{L}$ be a nest. If $\mathcal{L}$ is infinite, then alg $\mathcal{L}$ is inverse closed if and only if every atom of $\mathcal{L}$ is of finite dimension, and has order type $\alpha+\beta^{*}$, where $\alpha$ and $\beta$ are ordinals; If $\mathcal{L}$ is finite, then alg $\mathcal{L}$ is inverse closed if and only if every atom but one is finite dimensional.

\section{Spectra of operators in CSL ALgebras}

In this section, we discuss the properties of operators in a CSL algebra. We generalize results in [4] and [8].

Proposition 3.1. If $A \in$ alg $\mathcal{L}$, then $\sigma_{\mathcal{L}}(A) \subseteq \eta(\sigma(A))$, where $\eta(\sigma(A))$ denotes the full spectrum of $A$ in $B(H)$.

Proof. Let $\lambda_{0} \in \sigma_{\mathcal{L}}(A)$. Suppose that $\lambda_{0}$ is in the unbounded connected component of $\rho(A)$, the resolvent set of $A$. By Lemma 2.1, there exists an element $P \in \mathcal{L}$ such that $\left(\lambda_{0}-A\right) P H \subset P H$. So there exists a $\lambda$ in $\partial \sigma\left(\left.A\right|_{P H}\right) \cap \rho(A)$. This is impossible since

$$
\partial \sigma\left(\left.A\right|_{P H}\right) \subset \sigma_{\pi}\left(\left.A\right|_{P H}\right) \subset \sigma_{\pi}(A),
$$

where $\sigma_{\pi}$ is the approximate point spectrum.

We first give a proposition which will be needed in the sequel.

Theorem 3.2. Let $\mathcal{L}$ be a CSL. Then for every $A$ in alg $\mathcal{L}, \sigma_{\mathcal{L}}(A)=\sigma(A) \cup$ $\underset{\left\{E_{i}\right\}}{\cup}\left\{\cup_{i} \sigma_{\mathcal{L} E_{i}}\left(\left.E_{i} A\right|_{E_{i} H}\right):\left\{E_{i}\right\}\right.$ is a finite set of intervals of $\mathcal{L}$ with $\left.\sum_{i} E_{i}=I\right\}$.

In order to prove this proposition, we first give a lemma.

Lemma 3.3. Suppose that $\left\{E_{i}\right\}_{i=1}^{n}$ is a finite partition of $\mathcal{L}$, that is, each $E_{i}$ is an interval of $\mathcal{L}$ and $\sum_{i=1}^{n} E_{i}=I$. Then after appropriately arranging $\left\{E_{i}\right\}$, the matrix of each operators in alg $\mathcal{L}$ with respect to the decomposition $H=\sum_{i=1}^{n} \oplus E_{i} H$ is upper triangular.

Proof. Let $\mathcal{L}_{1}=\left\{P_{i}\right\}_{i=1}^{k}$ be a finite sublattice of $\mathcal{L}$ such that each $E_{i}$ is an interval of $\mathcal{L}_{1}$. For a fixed operator $A$ in alg $\mathcal{L}$, it is clear that $A$ has the form $\left(\begin{array}{cc}A_{11} & A_{12} \\ 0 & A_{22}\end{array}\right)$ with respect to $P_{1} H \oplus P_{1}^{\perp} H$. Since $I=P_{1}+P_{1}^{\perp}=P_{1}\left(P_{2}+P_{2}^{\perp}\right)+P_{1}^{\perp}\left(P_{2}+P_{2}^{\perp}\right)=$ $P_{1} P_{2}+P_{1} P_{2}^{\perp}+P_{1}^{\perp} P_{2}+P_{1}^{\perp} P_{2}^{\perp}$, the matrix of $A$ with respect to $H=P_{1} P_{2} H \oplus$ $P_{1} P_{2}^{\perp} H \oplus P_{1}^{\perp} P_{2} H \oplus P_{1}^{\perp} P_{2}^{\perp} H$ has the form

$$
\left(\begin{array}{cccc}
A_{11} & A_{12} & A_{13} & A_{14} \\
0 & A_{22} & A_{23} & A_{23} \\
0 & 0 & A_{33} & A_{34} \\
0 & 0 & 0 & A_{44}
\end{array}\right) .
$$

By induction, we can show that the matrix of $A$ with respect to $H=E_{1}^{\prime} H \oplus E_{2}^{\prime} H \oplus$ $\cdots \oplus E_{m}^{\prime} H$ has upper triangular form, where each $E_{i}^{\prime}$ is an atom of $\mathcal{L}_{1}$. Let $\bar{E}_{1}$ be the interval in $\left\{E_{i}\right\}$ which contains $E_{1}^{\prime}$. Deleting the atoms from $\left\{E_{1}^{\prime}, \cdots, E_{m}^{\prime}\right\}$ which are dominated by $\bar{E}_{1}$, let $\bar{E}_{2}$ be the interval in $\left\{E_{i}\right\}$ which contains the first remaining atom. By induction, we get a permutation $\left\{\bar{E}_{1}, \cdots, \bar{E}_{n}\right\}$ of $\left\{E_{i}\right\}$ in a 
similar way. It is not hard to see that every operator in alg $\mathcal{L}$ has upper triangular form with respect to $\bar{E}_{1} H \oplus \bar{E}_{2} H \oplus \cdots \oplus \bar{E}_{n} H$.

Proof of Theorem 3.2. It is clear that $\sigma_{\mathcal{L}}(A) \supset \underset{\left\{E_{i}\right\}}{\cup}\left\{\cup_{i} \sigma_{\mathcal{L} E_{i}}\left(\left.E_{i} A\right|_{E_{i} H}\right):\left\{E_{i}\right\}\right.$ is a finite set of intervals of $\mathcal{L}$ with $\left.\sum_{i} E_{i}=I\right\}$. We need to prove the converse. Without loss of generality, we suppose that $0 \in \mathbb{C}-\cup_{\left\{E_{i}\right\}}\left\{\cup_{i} \sigma_{\mathcal{L} E_{i}}\left(\left.E_{i} A\right|_{E_{i} H}\right):\left\{E_{i}\right\}\right.$ is a finite set of intervals of $\mathcal{L}$ with $\left.\sum_{i} E_{i}=I\right\} \cup \sigma(A)$. Let $\mathcal{L}_{1}$ be any finite sublattice of $\mathcal{L}$ with atoms set $\left\{E_{i}\right\}_{i=1}^{n}$. Then $A_{i i}$ is invertible in alg $\mathcal{L} E_{i}$ for each $i$. Let its inverse be $A_{i i}^{-1}$. By Lemma 3.3, we can suppose that the matrix of each operator in $\operatorname{alg} \mathcal{L}$ has upper triangular form with respect to $E_{1} H \oplus E_{2} H \oplus \cdots \oplus E_{n} H$. Put $B=A_{11}^{-1} \oplus A_{22}^{-1} \oplus \cdots \oplus A_{n n}^{-1}$. We have $B \in \operatorname{alg} \mathcal{L}$. Since $A B-I$ is strictly upper triangular, $N=A B-I$ is nilpotent in alg $\mathcal{L}$, that is, $N^{n}=0$. Therefore, $A B=N+I$ is invertible in alg $\mathcal{L}$ and so is $A$. This shows that $0 \notin \sigma_{\mathcal{L}}(A)$.

A well-known result of Ringrose [8] (or see [2]) is that, for any compact operator $K$, there is a maximal nest $\mathcal{N}$ such that $K \in \operatorname{alg} \mathcal{L}$ and $\sigma(K)=\sigma_{\mathcal{N}}(K)=\{0\} \cup$ $\left\{\sigma\left(\left.A_{\alpha} K\right|_{A_{\alpha} H}\right):\left\{A_{\alpha}\right\}\right.$ is the set of all atoms of $\left.\mathcal{N}\right\}$. The analogue is valid for CSL algebras which is actually implied in [5]. Here we give a direct proof.

Theorem 3.4. Let $K$ be a compact operator in alg $\mathcal{L}$ for a $C S L \mathcal{L}$, and let $G$ be the set of all atoms of $\mathcal{L}$. Then $\sigma_{\mathcal{L}}(K)=\sigma(K)=\{0\} \cup\{\underset{E \in G}{\cup} \sigma(E A E)\}$.

Proof. By proposition 3.1, $\sigma(K)=\sigma_{\mathcal{L}}(K)$. We only need to show the second equality. It is easy to see that $\delta(K)=\sum_{k} E_{k} K E_{k}$ is compact and $\sigma_{\mathcal{L}}(K) \supseteq$ $\cup_{E \in G}\left\{\sigma\left(\left.E K\right|_{E H}\right)\right\}$ by Theorem 3.2.

Let $G$ be the set of all atoms of $\mathcal{L}$. It is clear that

$$
\delta(K)=\sum_{E \in G} E K E
$$

is a compact operator. For a finite sublattice $\mathcal{F}$ of $\mathcal{L}$, let

$$
\mathcal{D}_{\mathcal{F}}(K)=\sum_{P \in \mathcal{F}} \triangle(P) K \triangle(P),
$$

where $\triangle(P)=P-\sup \{Q \in \mathcal{F}: Q<P\}$. By Theorem 5.4 of $[5], \mathcal{D}_{\mathcal{F}}(K) \rightarrow \delta(K)$ as $\mathcal{F} \rightarrow \mathcal{L}$. For any $\epsilon>0$, by the compactness of $\delta(K)$, there exist finitely many elements $E_{1}, \cdots, E_{n}$ in $G$ such that $\|E K E\|<\frac{\epsilon}{2}$ for every $E$ in $G \backslash\left\{E_{1}, \cdots, E_{n}\right\}$. Choose a finite sublattice $\mathcal{F}$ such that $\left\{E_{1}, \cdots, E_{n}\right\} \subset\{\triangle(P): P \in \mathcal{F}\}$ and

$$
\left\|\sum_{P \in \mathcal{F}} \triangle(P) K \triangle(P)-\delta(K)\right\| \leq \frac{\epsilon}{2} .
$$

Hence, for each $P^{\prime} \in \mathcal{F}$, either $P^{\prime}$ is an atom of $\mathcal{L}$ or

$$
\begin{aligned}
& \left\|\triangle\left(P^{\prime}\right) K \triangle\left(P^{\prime}\right)\right\| \leq\left\|\sum_{\substack{P \in \mathcal{F} \\
\Delta(P) \neq E_{1}, \cdots E_{n}}} \triangle(P) K \triangle(P)\right\| \\
& \leq\left\|\sum_{P \in \mathcal{F}} \triangle(P) K \triangle(P)-\delta(K)\right\|+\left\|\sum_{\substack{E \in G \\
E \neq E_{1}, \cdots E_{n}}} E K E\right\| \\
& <\frac{\epsilon}{2}+\frac{\epsilon}{2}
\end{aligned}
$$


For a fixed $\lambda \neq 0$, let $\epsilon=\frac{|\lambda|}{2}$. So there is a finite sublattice $\mathcal{F}_{0} \subset \mathcal{L}$ such that $\|\triangle(P) K \triangle(P)\|<\epsilon$ or $\triangle(P)$ is an atom of $\mathcal{L}$ for each $P$ in $\mathcal{F}_{0}$. If $\lambda \notin \sigma\left(\left.E K\right|_{E H}\right)$ for every $E \in G$, then by the choice of $\epsilon, \triangle(P)(\lambda-K) \triangle(P)$ is invertible in alg $\mathcal{L} \triangle(P)$ for every $P \in \mathcal{F}_{0}$. As in the proof of Theorem 3.2, we have $\lambda \notin \sigma_{\mathcal{L}}(K)$. Thus

$$
\sigma_{\mathcal{L}}(K) \subseteq \underset{E \in G}{\cup} \sigma(E K E) .
$$

In [4], it is proved that if $\mathcal{L}$ is a nest which has no infinite-dimensional atom, then $T \in \operatorname{alg} \mathcal{L}$ is invertible in $\mathcal{L}$ if and only if $T$ is invertible in $\operatorname{alg} \mathcal{L}+K(H)$. The analogue holds for a CSL algebra.

Theorem 3.5. Let $\mathcal{L}$ be a CSL on $H$ which has no infinite-dimensional atom, and let alg $\mathcal{L}+K(H)$ be closed. Then $T \in$ alg $\mathcal{L}$ is invertible if and only if it is invertible in alg $\mathcal{L}+K(H)$.

Proof. We need only to prove that if $T$ is invertible in alg $\mathcal{L}+K(H)$, then $T$ is invertible in $\operatorname{alg} \mathcal{L}$ for every $T$ in $\operatorname{alg} \mathcal{L}$.

Let $T^{-1}=A+K$ for some $A \in \operatorname{alg} \mathcal{L}$ and $K \in K(H)$. Then $T A=I-T K$ implies that $T K=I-T A \in \operatorname{alg} \mathcal{L}$ and $T K$ is compact. If $(I-T K) P H=P H$ for some $P$ in $\mathcal{L}$, then $T A P H=P H$ and hence $T P H=P H$ for $T^{-1} P H=A P H \subseteq P H$. So we suppose that $(I-T K) P H \subset P H$ for some $P$ in $\mathcal{L}$, that is, $1 \in \sigma_{\mathcal{L}(T K)}$.

Since $T K$ is compact and in alg $\mathcal{L}$, by Theorem 3.4, there are only finitely many atoms $E_{1}, E_{2}, \cdots, E_{n}$ of $\mathcal{L} P$ such that $\left.E_{i}(I-T K)\right|_{E_{i} H}$ is not invertible (note that $T K$ is compact), We first prove that, for any interval $E$ of $\mathcal{L} P$ with $E E_{i}=0$ for each $i, E T E H=E H$. As in the proof of Theorem 3.4, since $T K$ is compact there are finitely many intervals $F_{1}, F_{2}, \cdots, F_{m}$ of $\mathcal{L} E$ such that $E=F_{1}+F_{2}+\cdots+F_{m}$ and $\left.F_{i}(I-T K)\right|_{F_{i} H}$ is invertible in alg $\mathcal{L} F_{i}$. Therefore we can prove that $\left.E T A\right|_{E H}$ is invertible in $\operatorname{alg} \mathcal{L} E$ as in Theorem 3.2. So $\left.E T\right|_{E H}$ is invertible in $\operatorname{alg} \mathcal{L} E$, and then $E T E H=E H$.

Let $P_{i}$ be the smallest projection in $\mathcal{L}$ such that $P_{i} E_{i}=E_{i}$, and let $Q_{i} \in \mathcal{L} P$ be such that $E_{i}=P_{i}-Q_{i}$ for each $i$. If $P_{j} E_{i}=E_{i}$ for some $i \neq j$, then by the choice of $P_{j}, Q_{j}$, we have $Q_{j} E_{i}=E_{i}$ and then $P_{j}>Q_{j}>P_{i}>Q_{i}$. Without loss of generality, we assume that $Q_{1}<P_{1}<\cdots<Q_{n}<P_{n}$. So $\left.T\right|_{Q_{1} H}$ is invertible in alg $\mathcal{L} Q_{1}$ by the previous paragraph. Since $E_{1}$ is of finite dimension, $T P_{1} H=P_{1} H$ by Lemma 2.2 . Since $\left(Q_{2}-P_{1}\right) E_{i}=0$ for each $i$, it follows that $\left.\left(Q_{2}-P_{1}\right) T\right|_{\left(Q_{2}-P_{1}\right) H}$ is invertible in $\operatorname{alg} \mathcal{L}\left(Q_{2}-P_{1}\right)$ by the above paragraph. Hence, it is not hard to prove that $\left.T\right|_{Q_{2} H}$ is invertible in alg $\mathcal{L} Q_{2}$ and $T Q_{2} H=Q_{2} H$. By induction, $\left.E T E\right|_{E H}$ is invertible in alg $\mathcal{L} E$ for every interval $E$ of $\mathcal{L}$; in particular, $\left.T\right|_{P H}$ is invertible in $\operatorname{alg} \mathcal{L} P$. Therefore, by the arbitrariness of $P, T$ is invertible in $\operatorname{alg} \mathcal{L}$. The proof is complete.

Remark. When $\mathcal{L}$ has no atoms and alg $\mathcal{L}+K(H)$ is closed, for example, $\mathcal{L}\left(2^{\infty}, \leq, M_{p}\right)$ (see [6]), the result of Theorem 3.5 is valid clearly. When $\mathcal{L}$ has infinite dimensional atoms, the conclusion does not hold generally.

\section{ACKNOWLEDGEMENT}

The author would like to thank D. K. Davidson for some valuable suggestions. He also thanks Prof. Jipu Ma for his careful guidance. 


\section{REFERENCES}

1. K.R. Davidson, Commutative subspace lattices, Indiana Unive. Math. J. 27(3) (1978), 479490. MR 58:2340

2. K.R. Davidson, Nest algebras, vol. 191, Pitman Research Notes in Math., Longman Scientific and Techical, 1988. MR 90f:47062

3. K.R. Davidson, V.I. Paulsen and S.C. Power, Tree algebras, semi-discreteness, and dilation theory, Proc. London Math. Soc. 68(3) (1994), 178-202. MR 94m:47087

4. A. Feintuch and A. Lambert, Invertibility in nest algebras, Proc. Amer. Math. Soc. 91(4) (1984), 573-576. MR 85h:47049

5. E. G. Katsoulis, Integration with respect to a commutative subspace lattice, J. Operator Theory 22 (1989), 307-323. MR 91c:47071

6. E. G. Katsoulis and S. C. Power, Compact perturbations of certain CSL algebras, Proc. Amer. Math. Soc. 114(4) (1992), 1041-1045. MR 92g:47058

7. D. R. Larson, Triangularity in operator algebras, Pitman Research Notes in Math. In surveys of some recent results in operator theory, vol. 2, Longman Scientific and Technical, 1988, pp. 121-189. MR 90b:47002

8. K. R. Ringrose, Superdiagonal forms for compact linear operators, Proc. London Math. Soc. 12(3) (1962), 367-384. MR 25:458

Department of Mathematics, Nanjing University, Nanjing 210008, People's Republic OF CHINA

Current address: Post and Telecommunication Institute of Nanjing, Nanjing, 210003, People's Republic of China 